\title{
O Paradoxal Efeito Hydra em Populações: Aumento do Número de Indivíduos enquanto Aumenta a Taxa de Mortalidade.
}

\author{
Grace H. Conceicão \\ Depto de Biologia e Zootecnia, Feis, UNESP \\ 15385-000, Ilha Solteira- SP \\ Email: hingridberg@yahoo.com.br

\section{Berenice C. Damasceno Luciano Barbanti} \\ Depto de Matemática, Feis, UNESP \\ 15385-000, Ilha Solteira- SP

\section{RESUMO}

O fenômeno descrito como "efeito hydra" (onde hydra se refere ao ser mitológico que tendo a cabeça decepada outras aparecem em maior quantidade) permeia o estudo do crescimento populacional em ecologia.

É um consenso geral, que o aumento da taxa de mortalidade em uma população faz diminuir o seu tamanho ao longo do tempo. Como mostrado em [6] e [3], isto é verdade em todo o modelo do tipo não estruturado continuo de crescimento, densidade-dependente, em um meio constante.

Na década de 1950 e mais intensamente na década de 1970 alguns modelos particulares em que este consenso é contradito aparecem em [5], e recentemente uma visão mais abarcante e envolvendo o efeito hydra no crescimento populacional foi considerada em [1] , [2], [4].

Neste trabalho apresentamos um modelo de equação dinâmica discreta de crescimento populacional dada em [2] dependente de parâmetros reais e funções.

Damos condições sobre estes parâmetros e estas funções para que o efeito hydra apareça.

Seja: $k_{0} \in \mathbb{R}$.A equação à diferença que representa a variação populacional neste trabalho é dada como em [2], recursivamente por:

$x_{n+1}=g\left(k_{0}\right) x_{n} f\left(g\left(k_{0}\right) x_{n}\right)$,

para $k_{0}$ fixado onde $x_{n}$ é o tamanho da população no tempo $t_{n}, n \in \mathbb{N}$, com

$t_{0}>t_{1}>\cdots t_{m-!}>t_{m}>\cdots$, e $f, g: \mathbb{R} \rightarrow \mathbb{R}$ são duas funções apropriadas no

sentido que veremos à seguir no resultado principal deste trabalho. 
Teorema :Sejam $g: \mathbb{R}^{+} \rightarrow(0,1)$ uma função decrescente, $f: \mathbb{R}^{+} \rightarrow \mathbb{R}^{+}$diferenciável com $f^{\prime}<0$. Então, se para cada $k_{0}$ fixo, vale $f(x)>\frac{1}{g\left(k_{0}\right)}$, para todo $x \in \mathbb{R}^{+}$, então temos em (1) o efeito hydra.

Prova: De (1) temos que

$$
\frac{x_{n+1}}{x_{n}}=g\left(k_{0}\right) f\left(g\left(k_{0}\right) x_{n}\right)
$$

Como $f^{\prime}(x)<0$ para todo $x \in \mathbb{R}^{+}$e $g\left(k_{0}\right)<1$, vale

$f\left(g\left(k_{0}\right) x_{n}\right)>f\left(x_{n}\right)$.

Mas, por hipótese

$f\left(x_{n}\right)>\frac{1}{g\left(k_{0}\right)}$

Deste modo, por (3) e (4),vale

$f\left(g\left(k_{0}\right) x_{n}\right)>\frac{1}{g\left(k_{0}\right)}$

Observando (2) concluímos:

$\frac{x_{n+1}}{x_{n}}>1$

e assim

$x_{n+1}>x_{n}$,

acarretando o efeito hydra como explicitado acima, visto que

$$
g\left(k_{0}\right)<1
$$

e $f$ é decrescente.

Para exemplificar o resultado do Teor.1 vamos considerar na equação (1):

$g(k)=\frac{1}{k+1,5} \quad, \quad k_{0}=1, f(x)=\frac{3 x+1}{x} \quad, \quad x_{0}=3.000 \quad$,

Então a tabela do número de elementos da população no tempo é 


\begin{tabular}{|c|c|}
\hline TEMPO $\mathrm{t}_{\mathrm{n}} \mathrm{n}=$ & NÚMERO DE INDIVÍDUOS $\mathrm{x}_{\mathrm{n}}$ \\
\hline 0 & 3.000 \\
\hline 1 & 3.601 \\
\hline 2 & 4322 \\
\hline 3 & 5.188 \\
\hline 4 & 6.226 \\
\hline 5 & 7.472 \\
\hline
\end{tabular}

Conclusão: $O$ efeito hydra é considerado no âmbito de trabalhos em Ecologia como um paradoxo [2]. Vimos que há ao menos uma explicação "teórica" matemática para o fenômeno. $\mathrm{Na}$ área de Biologia/Ecologia podemos aproveitar este conhecimento encontrando uma população que se desenvolve segundo a lei em (1) com funções $\mathrm{f}$ e $\mathrm{g}$ que satisfaçam as condições do teorema 1.È o que estaremos investigando a seguir.

\section{Referências}

[1] P.A. Abrams, C. Quince. The impact of mortality on predator population size and stability in systems with stage structured prey. Theor. Pop. Biol., 68, 253-266, (2005).

[2] P. A. Abrams, When does greater mortality increase population size? The long history and diverse mechanisms underlying the hydra effect. Ecology Letters, 12:464-474, (2009).

[3] A. Hastyings. Population biology: Concepts and models, Springer, NY,(1997).

[4] E. Liz. Bubbles, chaos, and the hydra effect in delayed populational models. Colloquium on Diff. Eq. and Integration Theory, Krystni CZ, Masarykova Un. Press, p. 46, 2010.

[5] J. Maynard Smith. Models in Ecology, Cambridge University Press, Cambridge, UK, 1974.

[6] H. Seno. A paradox in discrete single species population dynamics with harvesting/thinning. Math.Biosci., 214, 63-69 (2008). 